\title{
Metal stent for refractory post-ES bleeding: Is this the ultimate treatment modality?
}

Authors

Institutions
Eisuke Iwasaki ${ }^{1}$, Takao $^{\text {Itoi }}{ }^{2}$, Takanori Kanai ${ }^{1}$

Keio University School of Medicine - Internal Medicine, Tokyo, Shinjukuku, Japan

${ }^{2}$ Tokyo Medical University - Department of Gastroenterology and Hepatology, Tokyo, Japan submitted

7. September 2016 accepted after revision 13. September 2016

\section{Bibliography}

DOI http://dx.doi.org/

10.1055/s-0042-117633

Published online: 24.11.2016

Endoscopy International Open

2016; 04: E1265-E1266

(c) Georg Thieme Verlag KG

Stuttgart · New York

E-ISSN 2196-9736

\section{Corresponding author}

Takao Itoi, MD, PhD,

\section{FASGE, FACG}

Department of

Gastroenterology

and Hepatology

Tokyo Medical University

6-7-1 Nishishinjuku

Shinjuku-ku

Tokyo 160-0023

Japan

Phone: +81-(3)-3342-6111

Fax: +81-(3)-5381-6654

itoi@tokyo-med.ac.jp
Endoscopic sphincterotomy (ES), which had been developed by Kawai [1], Classen [2], and Soma et al. [3], has been a very innovative and effective endoscopic procedure to date. However, ES has also inevitable post-procedural bleeding complications, the so-called "Achilles Hills of ES." Mirjalili et al. [4] previously demonstrated the distributions of the arterial supply of the major duodenal papilla and their relevance to ES according to their pathologic analysis of 19 cadaver pancreaticoduodenal specimens. Most of the arteries were related to the antero-superior and postero-inferior quadrants of the major duodenal papilla, both at their point of entry into the duodenal wall or bile/pancreatic ducts (microdissection) and in their distribution $5 \mathrm{~mm}$ from the tip of the papilla (histology) [4]. Moreover, the combined 10 and 11 o'clock segments of the papilla (as viewed endoscopically) contained only $10 \%$ and $11 \%$ of all papillary arteries on microdissection and histology, respectively, with seven of 19 specimens having no arteries in this region. Based on their results, they advocated that arterial bleeding as a complication of ES might be reduced by incising the papilla in the $10-11$ o'clock region rather than in the currently recommended 11-1 o'clock position. However, the axis of the real major papilla is often rotatable, mainly counter-clockwise.

Furthermore, a special anatomic situation (e.g., periampullary diverticula) causes confusion about the right axis of the major papilla. Thus, even if endoscopists believe that the 12 o'clock position is at the major papilla, this is not always true. These factors may lead to a wrong direction towards the papilla, causing post-ES bleeding. Thus far, large prospective studies have in fact reported that the incidence of active bleeding or ongoing oozing requiring endoscopic hemostasis ranges from $0.76 \%$ to $2.0 \%$ [5-7].

Several traditional techniques of endoscopic hemostasis for post-ES bleeding exist, namely, balloon tamponade, local injection of hypertonic saline-epinephrine, and thermal therapies such as argon plasma coagulation, bipolar coagulation, and/or clipping [8]. However, there is no consensus regarding the optimal endoscopic hemostatic technique for post-ES bleeding. Monotherapy or combination endotherapies produce very high rates of success for hemostasis, particularly in case of repeat therapies, but there are rare or only a few cases in which a conventional hemostatic technique is refractory. Traditionally, interventional radiology (arterial embolization) or surgical hemostasis is necessary.

Shah et al. [9] and Itoi et al. [10] previously introduced use of a fully covered self-expandable metal stent (FCSEMS) as a new hemostasis technique for refractory post-ES bleeding. This technique presents a new concept of hemostasis achieved by mechanical compression using FCSEMS. In fact, previous data showed a very high success rate without any serious complications $[9,10]$. Most recently, Cochrane and Schlepp [11] conducted a retrospective non-randomized controlled comparative study between the FCSEMS group ( $n=23$ ) and the non-FCSEMS group $(n=44)$ in patients with post-ES bleeding after primary endoscopic intervention failure particularly in patients with a high risk of post-ES bleeding. Interestingly, although the FCSEMS treatment group had a significantly lower bleeding rate at 72 hours (0.66g/dL vs. $1.98 \mathrm{~g} / \mathrm{dL} ; P<0.001)$, an increased proportion of patients with a high risk of bleeding ( $40 \%$ vs. $9 \% P=0.008$ ), and a significantly increased proportion of patients with moderate severity of bleeding events ( $52 \%$ vs. $9 \% P=0.0002$ ) were observed compared with the non-FCSEMS treatment group. The FCSEMS treatment group consisted of 9 patients with increased risk of bleeding but none had delayed bleeding compared with the 4 patients in the non-FCSEMS group, all of whom had increased risk of bleeding risk and developed delayed bleeding. Based on their results, they concluded that FCSEMS can provide homeostasis after 


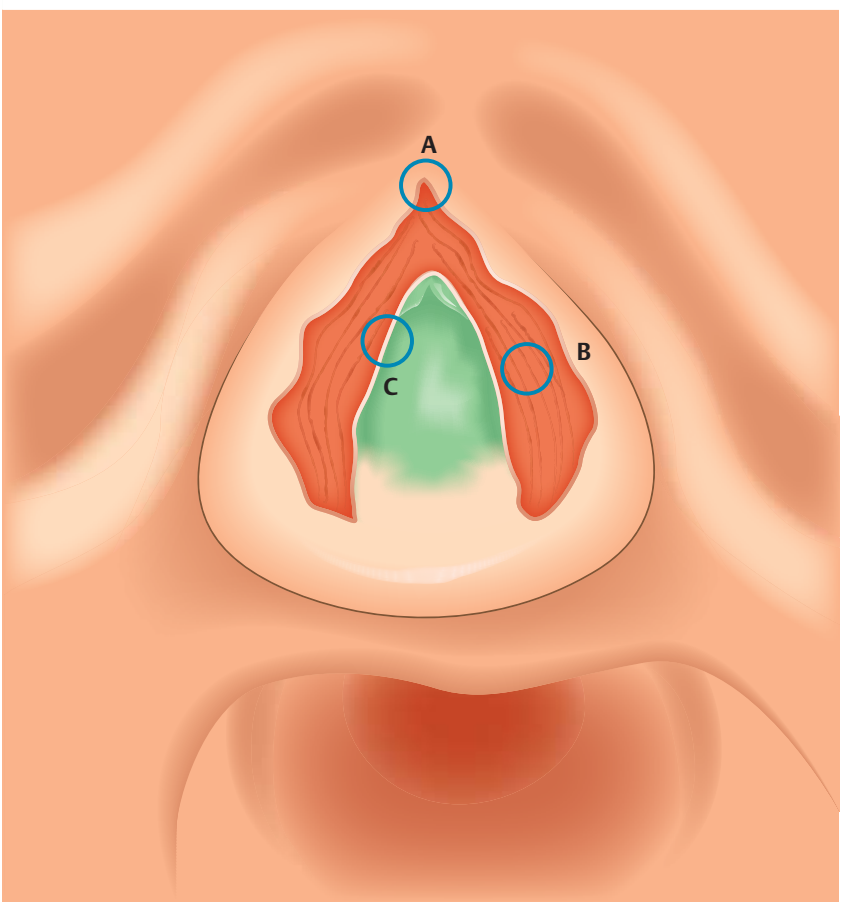

Fig. 1 Representative bleeding points after endoscopic sphincterotomy. a Maximum upper oral side of cutting area. $\mathbf{b}$ Papillary wall of cutting area. c Bile duct of cutting area.

primary endoscopic intervention failure and reduce the need for high-risk procedures.

Is FCSEMS placement suitable for all patients with refractory post-ES bleeding? Previous reports in the literature did not provide a clear explanation on this point. We presume that it depends upon the anatomic bleeding point. Post-ES bleeding is well known to originate largely from the incision area of the major papilla after ES (points A, B, and C in $\bullet$ Fig. 1) except for bleeding from the duodenal mucosa far from the cut area although rarely. All of these points appear suitable for FCSEMS placement. In our experience to date, complete hemostasis has been achieved at points $B$ and $C$. As far as point $A$ is concerned, however, re-bleeding was observed owing to incomplete compression. We infer that FCSEMS may be insufficient to compress the bleeding point at the most oral side in case of a relatively large ES incision, hence it may not be useful in this situation. In such cases, an additional conventional hemostasis technique after FCSEMS placement appears to be helpful.

Stent diameter and stent length are usually not controversial aspects of stent usage. However, theoretically, from the viewpoint of hemostasis using mechanical compression, FCSEMS with a stronger radial force or a larger diameter $(10 \mathrm{~mm})$ compared with the original bile duct and expanded biliary orifice appears to be suitable. In terms of stent length, a longer stent $(\geq 6 \mathrm{~cm})$ should be suitable to avoid migration into the bile duct. In fact, several reports have described a high ratio of stent migration (14.2\%; 3 in 21 patients) $[9,10,12,13]$. Regarding the type of metal stent, namely, uncovered/partially covered/fully covered metal stent, although the type of stent depends on the preference of endoscopists in case of malignant diseases, FCSEMS is ideal in case of benign diseases because stent removal after hemostasis is possible.

There is currently no solid evidence about when to use or remove a FCSEMS placed for hemostasis. Our and previous reports suggest that FCSEMS should be placed after the refractory or ongoing bleeding using mono or combined endoscopic treatment. This should be reasonable because conventional endoscopic hemostasis techniques are more cost-effective than FCSEMS placement. However, this method appears to be useful as a rescue technique in case of massive bleeding or hemorrhage resulting from an undetected bleeding point. In terms of stent removal in particular, we have no definite information regarding the duration of stent placement in benign cases. Previous studies have described a relatively short duration of 1 week to 4 weeks $[9,10]$ although Cochran and Schlepp described a longer duration of 88 days.

Care should be taken regarding procedure-related adverse events. FCSEMS with a stronger radial force and a large diameter may cause acute pancreatitis owing to the obstruction of the pancreatic duct orifice. We have encountered 3 cases in which acute pancreatitis occurred by the same mechanism. In such cases, we removed the FCSEMS the following day. Fortunately, hemostasis was achieved in all cases. Based on our experience, stent removal appears to be possible in just 1 day after FCSEMS placement.

In conclusion, the recently published CEASE Study by Cochrane and Schlepp in 2016 shows the efficacy of FCSEMS placement as a hemostatic modality for post-ES bleeding in high-risk patients. Taken together, the indication of FCSEMS placement should be carefully discussed taking into account information regarding the location of the bleeding point and the patient's risk of post-ES bleeding. More extensive prospective studies and randomized controlled trials are needed to confirm the safety, efficacy, and long-term outcomes of FCSEMS placement for the treatment of post-ES bleeding.

\section{Competing interests: None}

\section{References}

1 Classen M. Choledocholithiasis: Steinextraktion aus dem Gallengang endoscopisch moglich. Med Tribune 1973; 27: 1-5

2 Kawai K, Nakajima $M$ et al. Preliminary report on endoscopical papillotomy. J Kyoto Pref Univ Med 1973; 82: 353 - 355

3 Soma S, Tachikawa I, Okamoto Yet al. Attempt of endoscopic sphincterotomy and extraction of residual biliary stone. [in Japanese with English abstract]. Gastroenterol Endosc 1974; 16: 446-452

4 Mirjalili SA, Stringer MD. The arterial supply of the major duodenal papilla and its relevance to endoscopic sphincterotomy. Endoscopy 2011; 43: $307-311$

5 Freeman ML, Nelson DB, Sherman $S$ et al. Complications of endoscopic biliary sphincterotomy. N Engl J Med 26 1996; 335: 909-918

6 Masci E, Toti G, Mariani A et al. Complications of diagnostic and therapeutic ERCP: a prospective multicenter study. Am J Gastroenterol 2001; 96: 417-423

7 Loperfido S, Angelini G, Benedetti G et al. Major early complications from diagnostic and therapeutic ERCP: a prospective multicenter study. Gastrointest Endosc 1998; 48: 1-10

8 Tsou Y-K. Treating delayed endoscopic sphincterotomy-induced bleeding: Epinephrine injection with or without thermotherapy. World J Gastroenterol 2009; 15: 4823

9 Shah JN, Marson F, Binmoeller KF. Temporary self-expandable metal stent placement for treatment of post-sphincterotomy bleeding. Gastrointest Endosc 2010; 72: 1274-1278

10 Itoi T, Yasuda I, Doi S et al. Endoscopic hemostasis using covered metallic stent placement for uncontrolled post-endoscopic sphincterotomy bleeding. Endoscopy 2011; 43: 369-372

11 Cochrane J, Schlepp G. Comparing endoscopic intervention against fully covered self-expanding metal stent placement for post endoscopic sphincterotomy bleed (CEASE Study). Endoscopy International Open 2016; 4: DOI 10.1055/s-0042-118227

12 Di Pisa M, Tarantino I, Barresi L et al. Placement of covered self-expandable metal biliary stent for the treatment of severe postsphincterotomy bleeding: outcomes of two cases. Gastroenterology Research and Practice 2010; 2010: 138748

13 Valats JC, Funakoshi N, Bauret $P$ et al. Covered self-expandable biliary stents for the treatment of bleeding after ERCP. Gastrointest Endosc 2013; 78: $183-187$ 